\title{
Modelos de transposición de las Directivas 2002/91/CEy 2010/31/UE “Energy Performance Building Directive" en los Estados miembros de la UE. Consecuencias e implicaciones
}

\author{
Transposition of the 2002/91/EC and 2010/31/EU \\ "Energy Performance Building Directive" in the \\ EU Members States. Consequences and implications \\ A. García-Hooghuis ${ }^{(*)}$, F. J. Neila(*)
}

\section{RESUMEN}

La Directiva 2002/91/CE (Energy Performance Building Directive - EPBD (1)), publicada por la Comisión Europea y refundida en la Directiva 2010/31/UE (2), obliga a los países de Europa a cumplir con ciertos requisitos mínimos de eficiencia energética en los edificios y sus instalaciones. En ella se exige que cada Estado miembro incorpore la Directiva como legislación propia, estableciendo las condiciones y sistemáticas necesarias con carácter nacional (3). En el presente trabajo se efectúa una recopilación de las normas de eficiencia energética en la edificación a nivel Europeo, analizándose las consecuencias e implicaciones de los diferentes modelos de transposición de la EPBD adoptados por cada país. Además, se describen de modo general las metodologías más extendidas a la hora determinar la eficiencia energética de los edificios, los vacíos legales que presenta la normativa y las posibilidades de mejora en determinadas áreas, como acreditación de profesionales o incentivos financieros.

760-13

Palabras clave: Eficiencia energética; Directiva 2002/91/CE; Directiva 2010/31/ UE; DEEE; transposición.

\section{SUMMARY}

The 2002/91/EC Directive (Energy Performance Building Directive - EPBD (1)), published by the European Commission and recast in the 2010/31/EU (2), requires European countries to meet certain minimum energy requirements in buildings and their systems. Each Member state is obliged to incorporate the Directive as national law(s), establishing the necessary conditions and procedures at a national level (3). In the present document a compilation of energy efficiency standards for buildings across Europe has been made, analyzing the consequences and implications of the different transposition models of the EPBD adopted by each country. Furthermore, the methodologies most widely used for determining the energy efficiency of buildings, legal gaps and the possibilities for improvement in areas such as professional accreditations or financial incentives are also described.

Keywords: Energy efficiency; 2002/91/ EC Directive; 2010/31/EU Directive; EPBD; transposition.

(*) Escuela Técnica Superior de Arquitectura-Universidad Politécnica de Madrid, (España). Persona de contacto/Corresponding author: sandragarcia86@gmail.com (A. García-Hooghuis)

Recibido/Received: 01 mar 2012 Aceptado/Accepted: 24 nov 2012 Publicado online/ Published online: 11 jun 2013 


\section{INTRODUCCIÓN}

Teniendo en cuenta que en Europa los edificios son responsables de más del $40 \%$ del consumo energético (4) (5) y del $36 \%$ del total de emisiones de $\mathrm{CO}_{2}$ (6), es evidente que en la actualidad la edificación se ha convertido en un sector estratégico para cumplir con los objetivos del protocolo de Kyoto, del 20-20-20\% o de cualquier otro compromiso de reducción de emisiones y consumo energético. De hecho, según la Comisión de la Unión Europea, frente al sector del transporte y de la industria, son los edificios los que presentan el mayor potencial de ahorro de energía (7) y, tal como demuestran numerosos estudios, se podría llegar a recortar de manera rentable el consumo de energía en los edificios existentes entre un 20 y 50\% (8) más allá de los objetivos de reducción actuales, con un tiempo de recuperación de la inversión de 2 a 15 años gracias únicamente al ahorro económico que produciría la eficiencia energética derivada de la inversión (9). Por todo ello, la Comisión Europea publicó la Directiva 2002/91/CE del Parlamento Europeo y del Consejo del 16 de diciembre de 2002 relativa a la Eficiencia Energética de los Edificios (Energy Performance Building Directive-EPBD) (1) (conocida en España como DEEE) (1), refundida en la Directiva 2010/31/UE del 19 de Mayo de 2010 (2) en la que se obliga a los países de Europa a cumplir con una serie de requisitos mínimos de eficiencia energética en los edificios y sus instalaciones.

La EPBD es un instrumento jurídico que promueve un consumo eficiente de la energía en el sector de la edificación a nivel europeo, combinando instrumentos de carácter preceptivo (obliga a los Estados miembros a establecer requisitos de eficiencia energética para los edificios nuevos y existentes que sean objeto de reforma) e instrumentos de carácter informativo (tales como la emisión de certificados de eficiencia energética en el momento de la compra-venta o alquiler y los requisitos para las inspecciones de los sistemas de calefacción y aire acondicionado). En ella no se fijan de modo horizontal los niveles aplicables en todos los Estados miembros de la UE, sino que exige que cada uno de ellos incorpore la Directiva como legislación propia, determinando los requisitos y sistemáticas necesarios con carácter nacional (3). Es decir, obliga a los Estados miembros a establecer requisitos mínimos de eficiencia energética en los edificios y sus instalaciones, pero son los propios Estados miembros los que deciden cuáles son así como las metodologías de cálculo a aplicar. Este enfoque de tipo miembros, a la hora de fijar sus requisitos mínimos de eficiencia energética, tengan en cuenta sus características o condicionantes específicos, como la climatología o la construcción tradicional. Además permite a cada país elegir el alcance de sus metas de eficiencia energética, pudiendo incluso ir más allá de los requisitos mínimos establecidos en la Directiva (3). A pesar de estas ventajas, este planteamiento abierto tiene como inconveniente que ha desembocado en una situación normativa muy heterogénea, con el diseño de metodologías y sistemas significativamente diferentes dependiendo del Estado miembro de que se trate, e incluso con diferentes enfoques normativos para cada región de un Estado miembro, dificultando de modo notable el ejercicio de los profesionales dentro del sector, así como la comparación objetiva de datos y resultados de eficiencia energética de la edificación dentro de la UE.

En el presente trabajo se efectúa una recopilación de las normas de eficiencia energética en la edificación a nivel de la Unión Europea, identificando los organismos responsables, y se analizan las consecuencias e implicaciones de los diferentes modelos de transposición de la EPBD en los Estados miembros de la UE. Además, se describen de modo general las metodologías más extendidas a la hora determinar la eficiencia energética de los edificios en Europa, los vacíos legales que presenta la normativa y las posibilidades de mejora en determinadas áreas, como la acreditación de profesionales o el uso de incentivos financieros.

\section{MODELOS DE TRANSPOSICIÓN}

La aparición de la EPBD y su necesidad de transposición e implementación en función de la normativa y metodología previamente existentes en cada Estado miembro ha tenido consecuencias muy diversas en el marco legislativo de los diferentes países. En la Tabla $1^{*}$ se presenta la legislación en vigor en la que se han transpuesto ambas Directivas en los 27 Estados miembros de la UE, así como la normativa previa sobre la que se basan estas transposiciones, cuyo estudio de contenidos es fundamental para entender el modelo de transposición que se ha adoptado. También se identifican los organismos responsables encargados de su implementación y cumplimiento.

Según el modelo de transposición, los Estados miembros pueden agruparse en tres categorías:

- Estados miembros en los que la EPBD ha supuesto el primer enfoque a la normativa de construcción en cuanto a requisitos 
mínimos de eficiencia. Tal es el caso de Chipre, donde previamente a la aparición de la Directiva 2002/91/CE no existía ningún tipo de marco legislativo mediante el cual se regulasen los requisitos mínimos de eficiencia energética en la construcción, y es en estos países en los que la EPBD tiene mayor potencial de impacto. La transposición de la EPBD se ha realizado en base a las metodologías CEN desarrolladas específicamente para el cumplimiento de la Directiva, al no contar con normativa anterior que actuase como condicionante. Esto por un lado supone una ventaja en cuanto al proceso de homogeneización normativa progresiva en los países de la $U E$, pero por otro supone ciertas lagunas normativas, al no poder cubrir o enmendar las carencias de las normas CEN (descritas más adelante) mediante elementos de normativas anteriores.

- Estados miembros en los que sí existía normativa anterior en cuanto a eficiencia energética en la edificación, y en los que la transposición se ha realizado mediante enmiendas y órdenes de ejecución sobre el marco legislativo existente. En el caso de estos países se conservan las especificaciones, metodologías y procedimientos existentes en la normativa previa, pero se añaden las cláusulas que hacen referencia a la certificación energética de edificios, inspecciones y expertos. Este el caso en el que se encuentran la mayoría de Estados miembros.

- Estados miembros en los que la transposición de la EPBD ha dado lugar a un proceso de homogeneización o simplificación normativa. Tal es el caso de países como Austria, en la que previamente a la aparición de la EPBD existía una normativa específica en cuanto a códigos de construcción y requisitos mínimos para cada uno de sus 9 Länder. La implementación de la EPBD ha dado lugar a la aparición de una normativa común para todas las regiones, simplificando el marco legislativo a nivel nacional y facilitando la evaluación del impacto que supone el cumplimiento de los nuevos requisitos, al haberse puesto en común los objetivos, metodologías y metas de eficiencia energética a nivel nacional.

En cualquiera de los tres modelos de aproximación legislativa descritos anteriormente, la implementación de la EPBD ha influido de manera positiva en cuanto al endurecimiento de requisitos mínimos. Tanto en los países en los que existía normativa anterior como en los que había ausencia de ella, la EPBD ha supuesto un nuevo enfoque legal al establecimiento de requisitos mínimos de eficiencia energética en la edificación, marcando un camino común para toda la Unión Europea en cuanto al cumplimiento de objetivos de reducción de emisiones provenientes del sector de la edificación.

Como puede observarse en la Tabla 1*, existen dos aproximaciones a la aplicación de la legislación desarrollada a raíz de la aparición de la Directiva: la de ámbito regional y la de ámbito nacional. Así, existen Estados miembros en los que, en contraposición a los países que han desarrollado una normativa aplicable a nivel nacional, se ha optado por una normativa específica para cada zona, comunidad autónoma o región (como es el caso de España, Reino Unido o Italia, entre otros).

A su vez, existen dos modelos de esta aproximación de normativa regional. En el primero, como ocurre en el caso de Italia o España, se establece un tercer nivel en la jerarquía de competencias legales, permitiendo a las regiones o Comunidades Autónomas adaptar la normativa a sus necesidades dentro del marco legal que establece el reglamento a nivel nacional, desarrollado dentro del marco de la Directiva Europea. En el segundo, como ocurre en el caso de Reino Unido o Bélgica, se promulga una normativa independiente para cada una de sus regiones o provincias, casi a modo de países independientes. En este caso, aunque la normativa sea diferente para cada región, sin un marco legal a nivel nacional más allá del establecido en la EPBD a nivel europeo, pueden existir algunos puntos en común.

Como norma general, la implementación de la EPBD en los Estados miembros que han optado por este segundo sistema, es decir, una aproximación a nivel nacional (o regional, pero independiente para cada región) se encuentra en un estado más avanzado de desarrollo que aquellos Estados Miembros como España o Italia, que han optado establecer un marco legislativo a nivel nacional que deriva competencias a las Autonomías o Regiones. Se podría considerar que este modelo de aproximación regional tiene ventajas en cuanto a que permite una mejor adaptación de requisitos mínimos y procedimientos a las necesidades locales, pero en realidad los países que han adoptado normativas a nivel nacional también han tenido en cuenta las necesidades y particularidades locales con el establecimiento de, por ejemplo, diferentes zonas climáticas, para el cálculo de requisitos mínimos. La decisión del establecimiento de normativa a nivel nacional o regional, es por tanto una decisión política y no en pro de una mejor adaptación de requisitos y metodologías a las necesidades locales. 


\section{ORGANISMOS RESPONSABLES}

En cuanto a los organismos responsables de la implementación de la EPBD, los Estados miembros también han optado por varios modelos. En algún caso se ha asignado a un solo organismo la competencia de la implementación de la totalidad de su contenido, o como ocurre en la mayoría de Estados miembros, se ha decidido repartir competencias entre diferentes ministerios, siendo lo más habitual la separación de la responsabilidad de la implementación de la parte de certificación (artículos 3 a 7 de la EPBD 2002/91/CE) y la del contenido en materia de inspecciones (artículos 8 y 9). Además, en ocasiones incluso se han creado organismos específicos con motivo de la implementación de la EPBD (como el SEEB en Dinamarca).

Por otra parte, los organismos estatales (Ministerios, etc.) normalmente han delegado responsabilidades en otras entidades, sobre todo en ámbitos de garantía de calidad y formación y acreditación de expertos independientes.

\section{EL PAPEL DE LAS NORMAS CEN}

En referencia al artículo 3 de la EPBD 2002/91/CE, en el que se instaba a los Estados miembros a aplicar, a escala nacional o regional, una metodología de cálculo de la eficiencia energética de los edificios teniendo en cuenta las normas o regulaciones contenidas en el derecho interno de los propios Estados miembros, la Comisión Europea encargó al Comité Europeo de Normalización (CEN) el desarrollo de normas y metodologías de evaluación del rendimiento energético de la edificación (mandato M 343-EN).

Debido al corto intervalo de tiempo entre la fecha de emisión del mandato y la fecha límite para la implementación de la EPBD, y ya que las normas CEN continuaban en fase de desarrollo (la mayor parte de ellas se publicaron en los años 2007-2008), prácticamente todos los Estados miembros optaron por desarrollar su propia metodología de cálculo a nivel nacional para evaluar la eficiencia energética de la edificación. La progresiva incorporación de las normas CEN a la metodología propia de cada Estado miembro complica aún más la situación normativa dentro de la UE (10).

Las normas publicadas por el CEN establecen las especificaciones metodológicas básicas, pero la mayoría dejan abiertas opciones a escoger por los propios Estados miembros. Algunos aspectos, por su complejidad, ni siquiera se tratan en las normas CEN, como por ejemplo, la combinación de sistemas de iluminación natural y sombreamiento, cogeneración o sistemas de renovables integrados en la edificación tales como colectores para solar térmica, colectores fotovoltaicos, calderas de biomasa, turbinas eólicas, absorbentes higrotérmicos, sistemas de enfriamiento pasivo, construcciones de doble piel, etc. (11). Otro problema fundamental que presenta la normativa CEN es la dificultad de su aplicación en edificios existentes, derivada sobre todo de la obtención de los datos necesarios para poder realizar los cálculos correspondientes. Para solventar este problema muchos países han optado por desarrollar manuales de asistencia para certificadores en los que se detallan valores típicos para las propiedades de los materiales en componentes constructivos dependiendo de su antigüedad, así como factores de eficiencia de diferentes sistemas constructivos.

Entre las normas CEN más utilizadas de manera total o parcial por los Estados miembros, cabe mencionar:

- EN 15217: Energy performance of buildings-Methods of expressing energy performance and for energy certification of buildings.

- EN 15203: Energy performance of buildings-Assessment of energy use and definition of ratings.

- EN 15315: Energy performance of buildings-Overall energy use, primary energy and $\mathrm{CO}_{2}$ emissions.

- EN 15316: Heating systems in buildingsMethods for calculation system energy requirements and system efficiencies.

- EN 15243: Ventilation for Buildings-Calculation of room temperatures and of load and energy for buildings with room conditioning systems.

- EN 15193: Energy performance of buildings-Energy requirements for lighting.

- EN ISO 13790: Energy performance of buildings-Calculation of energy use for space heating and cooling.

- En 15241: Ventilation for buildings-Calculation methods for energy requirements due to ventilation systems in buildings.

- EN 15232: Energy performance of buildings-Impact of building automation, controls and building management.

- EN 15265: Energy performance of buildings-Calculation of energy needs for space heating and cooling using dynamic methods. General criteria and validation procedures.

- EN 15603: Energy performance of buildings-Overall energy use and definition of energy ratings.

- EN 15240: Ventilation for buildings-Energy performance of buildings. Guidelines for inspection of air-conditioning systems.

- EN 15239: Ventilation for buildings-Inspection of ventilation systems 
En la actualidad, siete Estados miembros han implementado metodologías de desarrollo propias, sin incluir los estándares descritos en las normas CEN, mientras otros, como Chipre, han optado por lo contrario: basar toda la metodología de cálculo, certificaciones e inspecciones en lo descrito en las normas CEN. La aproximación más común, y también la más acertada ya que deriva en metodologías y sistemas más completos y concretos, ha sido la que han adoptado la mayoría de Estados miembros, que consiste en una combinación de las normas CEN existentes con metodologías propias de cada Estado miembro. En este caso, como se ha descrito con anterioridad, se tiende la mano a un proceso de homogeneización normativa en el ámbito europeo y se suplen las carencias metodológicas que presentan las normas CEN en la actualidad mediante elementos adaptados a las particularidades o necesidades propias de cada país, lo cual es el objetivo último del modelo legislativo de la EPBD. En algunos países incluso se ha recurrido a normas internacionales ajenas a la EPBD como parte de la metodología.

\section{METODOLOGÍA DE CÁLCULO DEL RENDIMIENTO ENERGÉTICO DE LOS EDIFICIOS: CERTIFICACIÓN ENERGÉTICA}

En la Tabla $2 *$ se resume la metodología adoptada por cada Estado miembro para calcular la eficiencia energética de sus edificios así como los modelos o sistemas de representación de los resultados obtenidos. Se especifica además el número de escalas con diferentes valores umbral para cada tipología de edificio.

Los Estados miembros de la UE han desarrollado dos tipos de procedimientos para la clasificación energética de edificación. El primero y más extendido se basa en el cálculo de la demanda energética (o como define la EPBD en el anexo I, la cantidad calculada de energía consumida anualmente para satisfacer las distintas necesidades ligadas a su uso normal) mientras que sólo Suecia utiliza el segundo, basado en la medida del consumo como forma de clasificación (cantidad real de energía). Algunos países, como Alemania, Irlanda o Finlandia combinan ambos métodos para la clasificación energética de su edificación, dependiendo de la tipología del edificio.

La decisión de cada Estado miembro de clasificar energéticamente sus edificios en cuanto a valores de demanda (calculados) o de consumo (medidos) atiende en la mayoría de los casos a planteamientos políticos (12).
Cada uno de los métodos presenta sus pros y contras, que deben valorarse a la hora de elegir la opción que más se adecue a la situación particular de cada país. Así, por ejemplo, al certificar en base a la medida de consumo es muy complicado establecer umbrales estándar de clasificación del edificio, ya que se ha de tener en cuenta que el uso de energía en los edificios depende en gran medida de los hábitos de consumo de sus ocupantes, y el objetivo de la certificación energética es certificar un edificio y no a sus habitantes. Este problema, muy determinante en el caso de viviendas unifamiliares, no lo es tanto en grandes edificios residenciales, en los que un cambio en los hábitos de consumo de uno de sus ocupantes normalmente no repercute de manera demasiado significativa sobre el consumo total del edificio. Además, al certificar según la medida de consumo es muy complicado establecer hipótesis de ahorro energético potencial, ya que establecer una estimación del efecto de las medidas de ahorro energético para cada parte del edificio requeriría una subdivisión de consumo energético para las diferentes partes del mismo en el certificado (que no suele darse), o bien la colocación de contadores en cada zona a considerar.

El método de clasificación energética según la medida de consumo suele ser más barato que según el cálculo de la demanda, al requerir mucho menos tiempo la obtención de datos para establecer la eficiencia energética del inmueble. En este tipo de método la información obtenida acerca del comportamiento térmico de las fachadas y las instalaciones suele ser muy escasa. Por el contrario, el cálculo de la demanda ofrece la posibilidad de obtener información detallada acerca del comportamiento térmico de la envolvente y las instalaciones. Otra ventaja del cálculo de la eficiencia energética según la demanda es que al ser un método de cálculo estandarizado, siendo los parámetro de cálculo (las cargas y datos climáticos) también datos estándar, es posible comparar de manera objetiva e inmediata la eficiencia energética de dos edificios, lo que no era tan sencillo en edificios certificados mediante la medida de consumo. La desventaja principal de este método es que la obtención de información para poder realizar el cálculo de la demanda estableciendo un modelo de edificación apropiado, sobre todo en el caso de edificios antiguos que se han sometido a diversas remodelaciones, requiere un tiempo considerable. Teniendo en cuenta este factor tiempo, el coste económico de obtener un certificado de eficiencia energética usando la metodología basada en el cálculo de demanda es mayor que aquel en el que se ha utilizado el método de medida de consumo (12). 
1. Certificado de eficiencia energética en edificios residenciales según demanda (EnEV 2009) - Ejemplo de sistema de representación en barra continua (Alemania).

2. Building Energy Rating (BER). Building Amendment Regulatios SI 229/2008 - Ejemplo de sistema de representación escalonado (Irlanda).
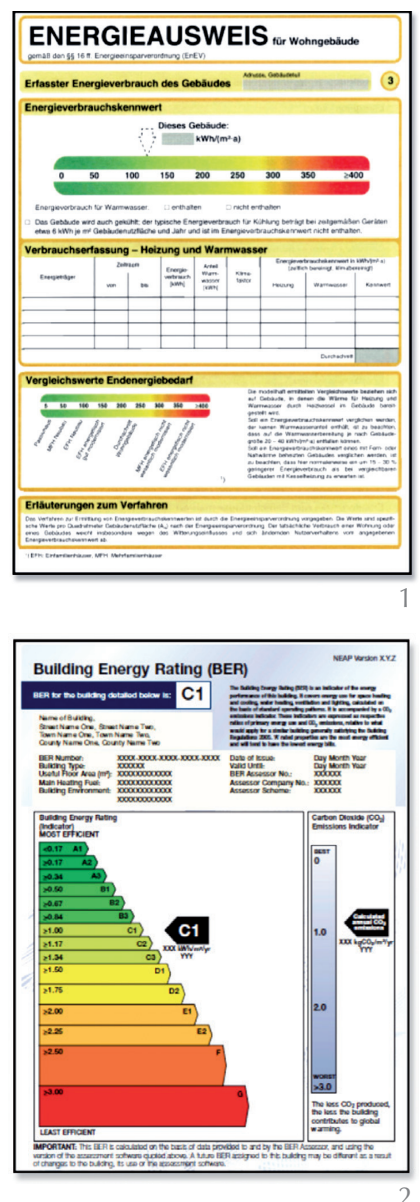

Por ello, algunos Estados miembros han optado por una u otra metodología dependiendo de las características del edificio, aunque este planteamiento es más costoso en términos normativos y económicos, y presenta el problema de que habría que establecer un sistema de comparación entre los cálculos de demanda y consumo en el mismo certificado.

El uso de uno u otro método según el tipo de edificio es correcto en cuanto a intenciones, al abaratar el coste de la certificación para algunas tipologías y, ya que ha de exponerse el correspondiente certificado de eficiencia energética en los edificios públicos, puede hacer más legibles o fácilmente entendibles los resultados al estar el público familiarizado con estas medidas de consumo. Sin embargo, la posibilidad de utilizar ambos métodos de cálculo y la consiguiente existencia de varios tipos de certificados se aleja de lo que debería de ser el fin último de la implementación de la EPBD: el establecimiento de un sistema de categorización energética común a todos los edificios, en el que pueda compararse de un modo directo la eficiencia energética de un edificio con otro. Debería por tanto existir, en el caso de los países que utilizan diferentes métodos de cálculo de eficiencia para distintas tipologías de edificios, una escala de valores equivalentes común a los resultados de uno u otro método, según ciertos coeficientes de equivalencia o de corrección.

Lo mismo ocurre en el caso de países que han adoptado diferentes escalas con valores umbral para cada categoría o tipología de edificio (hasta 29 escalas, como en el caso de Irlanda), en los que, a pesar de poder comparar con total precisión edificios de tipologías similares entre sí, es muy difícil para el usuario tener una visión global de la categoría de consumo en comparación con edificios de otras tipologías.

Este problema se solventa en países como Alemania, que ha establecido una única escala de valores para todas las tipologías en una barra continua, en la que se indica el consumo tipo de un edificio similar al que se evalúa. Se puede efectuar así una comparación en términos absolutos con las demás tipologías, pero también del propio edificio en relación a otros de su misma tipología. Es decir, marcando por ejemplo el consumo de un hospital en la parte de valores menos eficientes, se puede constatar que se trata de edificaciones con un gran consumo con respecto a otros tipos, como por ejemplo una vivienda, pero se puede comprobar también la eficiencia del hospital en cuestión con respecto a lo que sería la media de consumo energético de edificios de su misma tipología.
Además, este sistema en barra de valores continua común a todas las tipologías, facilita el modo de plasmar en el certificado el endurecimiento de requisitos mínimos según la revisión quinquenal prevista en el artículo 4, apartado 1, de la Directiva 2010/31/UE, ya que en la actualidad, en casi todos los Estados miembros existe un vacío normativo al respecto (Figuras 1 y 2 ).

Tampoco se ha definido con claridad en la mayoría de Estados miembros el modelo de certificación de edificios de usos mixtos. En algunos países, como Finlandia o Francia, se da la opción, igual que ocurre con los bloques de viviendas, de certificar bien por unidad o bien por edificios enteros. La solución adoptada en países como Irlanda o la región de Bruselas Capital dentro de Bélgica, es ofrecer la posibilidad de emitir varios certificados (uno por cada uso), o en el caso de certificarse el edificio completo, ponderar la eficiencia energética en un resultado que tenga en cuenta la superficie que ocupa cada uso en relación al total.

En cuanto a la certificación energética de edificios de nueva construcción, ésta debería realizarse a lo largo de la fase de proyecto (incluso en varios momentos de la misma) para que realmente pudiese tener impacto en el diseño de la edificación en términos energéticos. En la actualidad, en muchos países se certifica tras finalizar la construcción, momento en el que el único valor que tiene la certificación es la comprobación del cumplimiento de los requisitos mínimos. Para que la certificación energética de edificios de nueva construcción surtiese efecto en términos de eficiencia energética, debería plantearse como un valor añadido y no como un mero documento informativo, creando en todos los países, tal y como ya han hecho algunos, incentivos financieros a la construcción y diseño de edificios de clases de alta eficiencia energética.

\section{INCENTIVOS FINANCIEROS Y BARRERAS DE MERCADO}

El 14 de noviembre de 2012 se ha publicado la Directiva 2012/27/UE relativa a la eficiencia energética, que complementa la Directiva 2010/31/UE. En ella se establecen normas para eliminar barreras en el mercado de la energía y obligar a los Estados miembros a implantar una estrategia a largo plazo para movilizar inversiones en la renovación del parque inmobiliario nacional.

Para el correcto diseño de los programas de incentivos financieros ofrecidos por el Estado para la eficiencia energética en la edificación, es fundamental el registro estadístico de los datos obtenidos en el proceso 
de certificación. Los programas de subvenciones, créditos blandos, reducción de impuestos y demás incentivos a la eficiencia energética deberían destinarse a sectores estratégicos según las necesidades, características y carencias constructivas que existen en cada Estado miembro. La certificación energética de la edificación constituye una buena oportunidad para disponer de esos datos y analizar a fondo los factores de los que derivan, registrando en una base de datos la información obtenida en inspección y certificación y pudiendo disponer de ella para fines estadísticos en base a los cuales mejorar la metodología centrándose en los aspectos más necesarios en cada país. A pesar de ello, en este momento, sólo en torno a la mitad de los Estados miembros cuentan con un sistema de registro y análisis de datos de certificación energética.

\section{INSPECCIÓN DE LAS INSTALACIONES DE CALEFACCIÓN Y AIRE ACONDICIONADO}

Los diferentes tipos y métodos de inspección en los Estados miembros, así como la adopción de las opciones " $\mathrm{A}$ " (inspecciones) o "B" (campañas de información) para calderas y sistemas de calefacción que se contemplaban en el artículo 8 de la Directiva 2002/91/CE, derivan en una multitud de enfoques distintos en cada país. Además, por lo menos en 11 Estados miembros se han considerado otras Directivas de la UE adicionales a la EPBD que afectan a las inspecciones, como la Directiva 2005/32/ EC que establece un marco para los requerimientos de productos que usan energía, o la Directiva 2006/32/EC sobre los usos finales de la energía y servicios energéticos, o incluso se han tenido en cuenta los beneficios del uso de energías renovables de acuerdo a la Directiva 2009/28/EC, pudiendo aplicar también el sistema de inspecciones a calderas de biomasa (13).

Mientras que algunos Estados miembros han decidido implementar soluciones combinadas, mezclando en cierta forma campañas de información e inspecciones, como por ejemplo inspecciones o pre-inspecciones acompañadas de campañas de información hacia el público objetivo o campañas de información complementadas con esquemas de inspección voluntarios, la mayoría de Estados miembros se han decidido por una u otra opción, evaluando además algunos países la rentabilidad de la metodología escogida y midiendo el impacto en la eficiencia energética de la misma. Aproximadamente la mitad de los Estados miembros han escogido la opción A, y los restantes la opción B, fundamentando en la mayoría de los casos su decisión en los procedimientos ya existentes en el país antes de la aparición de la Directiva. En este aspecto, la flexibilidad de la EPBD ha permitido adaptar el enfoque de la metodología de inspección a los hábitos de consumo y modelos de distribución energética de cada Estado miembro: así en los países del sur se ha hecho más hincapié en el desarrollo de normativa en cuanto a inspecciones de aire acondicionado, mientras que en los Estados miembros del norte de Europa, la normativa en este aspecto está en su fase inicial de desarrollo, y en determinados países de Centroeuropa ha sido necesario adaptar la normativa de inspecciones a los modelos de redes de calor distritales, donde no existen prácticamente calderas individuales y en los que un sistema de inspecciones a este nivel no tendría mucho sentido.

En este momento, las inspecciones de calderas, aire acondicionado y certificación energética de edificios se tratan como tres temas independientes, existiendo normativas diferentes para cada uno y siendo incluso competencia de diferentes organismos en la mayoría de Estados miembros. Sería recomendable que todos los países incluyesen también en el proceso de certificación energética de la edificación, la inspección de sus instalaciones de calefacción y aire acondicionado, como ocurre en Suecia, ya que esto permitiría no sólo obtener una visión más global de la eficiencia energética del edificio y sus sistemas, sino también facilitar la sistemática en cuanto a normativa y formación y competencias de expertos independientes.

\section{EXPERTOS Y SISTEMAS DE CONTROL INDEPENDIENTES}

En la actualidad, en gran parte de Estados miembros, la administración estatal delega la acreditación y formación de expertos en otros organismos. Incluso en muchas ocasiones, estos autorizan a su vez a terceros a formar y a acreditar a expertos independientes en materia de certificación e inspecciones, lo que genera diferencias enormes en cuanto a contenidos de formación, sistemas y requisitos de acreditación dentro de un mismo Estado miembro, y dificulta además el ejercicio de profesionales en todo el territorio europeo. Además, no existe consenso en cuanto a las categorías de expertos independientes en cada país, dependiendo éstas de los modelos y metodologías de certificación para cada categoría de edificio en el país correspondiente. Mediante el estudio comparativo de la situación normativa en los diferentes Estados miembros se ha comprobado que en la actualidad, en un $44 \%$ de los Estados miembros es el mismo profesional acreditado el que lleva a cabo las certificaciones de edificios e inspecciones 
de sus instalaciones (una sola categoría de expertos), un $20 \%$ tiene dos categorías de expertos (una para certificaciones y una para inspecciones), y el $36 \%$ restante cuenta con 3 o más categorías de expertos.

Sobre todo en el caso de inspectores de instalaciones de calefacción y aire acondicionado, a menudo no es necesaria formación específica, ya que a raíz de la EPBD los profesionales que realizaban con anterioridad tareas de instalación y mantenimiento de sistemas han adquirido nuevas competencias, sin estar regulados dentro del marco normativo específico de la Directiva.

Haría falta, por tanto, construir un modelo de formación y ejercicio profesional común de expertos independientes a nivel europeo, homogeneizando criterios y competencias, y que permitiese además un correcto sistema de garantía de calidad, inexistente en este momento en numerosos Estados miembros.

En los países en los que existe un sistema de garantía de calidad, se han desarrollado dos modelos diferentes: el primero que permite el ejercicio de expertos independientes únicamente como parte de un organismo acreditado, y que sea éste el encargado de desarrollar su propio sistema de garantía de calidad, estableciendo auditorías externas, y el segundo que permite el ejercicio de los expertos independientes de manera individual, como autónomos, existiendo un sistema de garantía de calidad a nivel nacional. Para ello de nuevo sería necesario una base de datos o registro de certificados e inspecciones, que en este momento muchos países no tienen en funcionamiento. En ocasiones, se pide al propio experto independiente que conserve durante un periodo de 10 años todos los certificados que ha emitido para poder presentarlos a la autoridad en caso de requerirlo.

\section{CONCLUSIONES}

Un aspecto positivo de la EPBD es que ha supuesto en todos los Estados miembros un endurecimiento de requisitos mínimos de eficiencia energética, o un cambio en el modelo de aplicación de los existentes. Sin embargo, estos requisitos mínimos son aplicables únicamente a edificios de nueva construcción y a rehabilitaciones de edificios existentes. Teniendo en cuenta que el parque inmobiliario de nueva construcción representa una ínfima parte del total edificado en comparación con los edificios existentes (14), es evidente que el aspecto principal que debería de abordarse en la EPBD es el establecimiento de requisitos mínimos para edificios existentes.
Una forma en la que podría llevarse a cabo este objetivo sería obligar a la implementación de las recomendaciones de posibles mejoras de eficiencia energética que se emiten con los certificados. Todos los países han desarrollado sistemas para la emisión de recomendaciones, generadas en ocasiones de forma automática por el software, pero siendo en la mayoría de los casos el experto independiente el que las elabora de manera particularizada para cada edificio, invirtiendo tiempo del proceso de certificación en calcular el ahorro que podría suponer su implementación tanto en términos energéticos como económicos. Sin embargo, en la actualidad estas las recomendaciones sólo quedan registradas a título informativo, ya que prácticamente ningún Estado miembro establece plazos de obligatoriedad para la aplicación de las mismas. El establecimiento de clases energéticas mínimas no sólo para edificios de nueva construcción sino también para edificios existentes, obligando a realizar de manera progresiva las mejoras propuestas en los certificados, supondría un paso de gigante en cuanto a eficiencia energética en la edificación. Además, es en los edificios de más antigüedad donde las medidas son más efectivas en relación al coste de su implementación.

Para que lo anterior pudiese llevarse a cabo, sería también necesario el desarrollo de un sistema de sanciones por incumplimiento de requisitos mínimos de eficiencia energética y certificación que en la actualidad solo existe en la mitad de los Estados miembros.

El planteamiento de Directiva marco de la EPBD permite a los Estados miembros adaptar la normativa a sus necesidades concretas, aproximándose con el endurecimiento progresivo de los requisitos mínimos particulares de cada uno a un modelo de edificio de consumo casi nulo. Por un lado, esto supone una ventaja en cuanto a que permite aprovechar los recursos específicos de cada país para alcanzar el objetivo común de consumo nulo (por ejemplo energía solar en países del sur de Europa, o recogida de pluviales en el norte), pero por otra parte, permitir una libre aplicación de la norma dificulta mucho que los procesos se hagan de una forma correcta y que se alcancen resultados óptimos en el conjunto, generándose diferentes velocidades de implantación. Así, algunos Estados miembros han desarrollado estrategias a largo plazo para alcanzar los requisitos energéticos para edificios de consumo casi nulo descritos en el artículo 9 de la EPBD 2010/31/UE. Por ejemplo, en los Países Bajos se ha planteado reducir el consumo energético en comparación al actual un 50\% en 2015, y un 100\% en 2020 y Reino Unido se pretende que los 
edificios construidos en 2016 sean edificios de consumo casi 0 (15). En otros países, por el contrario, el nivel de compromiso es mucho menor. Un claro ejemplo es el caso de España, que ha sido denunciada el 24 de noviembre de 2011 ante el Tribunal de Justicia de la Unión Europea por la Comisión Europea por no haber tomado todavía las medias necesarias para la transposición de las especificaciones de la EPBD. En la actualidad, el Real Decreto por el que se aprobará el procedimiento básico para la certificación de eficiencia energética de edificios existentes está todavía en fase de proyecto.

Para que el planteamiento de Directiva marco fuese realmente eficaz, debería establecerse un modelo mediante el cual los países se adaptasen a la norma y no al contrario, como ocurre en la actualidad, en el que los Estados miembros amoldan la norma a sus necesidades específicas. Es decir, debería de plantearse un objetivo común de obligado cumplimiento, instituyendo fórmulas y metodologías comunes con cierta flexibilidad en lugar de permitir que sean los Estados miembros los que desarrollen sus propias metodologías, lo que ha generado resultados muy dispares. Por ejemplo, la normativa debería de ser común a todos los países en cuanto a aire acondicionado, estableciendo zona climáticas para los requisitos mínimos en cada país (igual que se ha hecho dentro de los propios países como en el caso de España o Francia). Esta aproximación es más complicada pero más efectiva, teniendo en cuenta el dinero y el tiempo invertido por los países en adaptarse o encontrar fórmulas propias. Las normas CEN deberían desarrollarse hasta convertirse en documentos marco que establezcan principios generales comunes para todos los Estados miembros, aún permitiendo una máxima flexibilidad a la hora de su aplicación (16).

En cualquier caso, aunque la EPBD ha supuesto un enorme avance como herramienta de mejora de la eficiencia energética de los edificios, con la consiguiente reducción las emisiones de $\mathrm{CO}_{2}$, es poco probable que estos objetivos se alcancen sin que exista una legislación más estricta, se disponga de sistemas de financiación eficaces y se logre que las personas involucradas en el sector de la edificación realmente tomen conciencia de la gravedad del problema y por tanto se comiencen a aplicar las medidas técnicas necesarias.

\section{REFERENCIAS}

(1) http://eur-lex.europa.eu/LexUriServ/LexUriServ.do?uri=OJ:L:2003:001:0065:0071:ES:PDF

(2) http://eur-lex.europa.eu/LexUriServ/LexUriServ.do?uri=OJ:L:2010:153:0013:0035:ES:PDF

(3) Comisión de las Comunidades Europeas COM (2008) 780 final-2008/0223 (COD)-Propuesta de Directiva del Parlamento Europeo y del Consejo relativa al rendimiento energético de los edificios (refundición) (presentada por la Comisión). Bruselas, 13.11.2008

(4) Green Building Impact Report. Greener buildings. Pew Center on Global Climate Change, noviembre 2008.

(5) Holl, M. (15-16 de noviembre, 2010). The new EPBD and its policy towards nearly zero energy buildings SBN Conference. Paris.

(6) Potencial de ahorro energético y de reducción de emisiones de $\mathrm{CO}_{2}$ del parque residencial existente en España en 2020. Informe WWF 2010

(7) Comisión Europea COM (2011) 109 final. Comunicación de la Comisión al Parlamento Europeo, al Consejo, al Comité Económico y Social Europeo y al Comité de las Regiones. Plan de Eficiencia Energética 2011. Bruselas, 8.3.2011.

(8) Roadmap 2050: a practical guide to a prosperous low-carbon Europe. European Carbon Foundation. Abril 2010

(9) Sweatman, P., Managan, K. (2010). Financing Energy Efficiency Building Retrofits. International Policy and Business Model Review and Regulatory Alternatives for Spain. Climate \& Strategy Partners, Madrid.

(10) Erhorn, H., Erhorn Kluttig, H. (2011). Procedures (Core Theme 4) Concerted Action. Energy Performance of Buildings. Bruselas.

(11) Detailed report on procedures for energy performance characterisation-Concerted Action. Supporting transposition and implementation of the directive 2002/91/EC-EPBD (2005-2007) CIP y IEE 2008.

(12) Engelund Thomsen, K., Wittchen, K.B. (2011). Certification (Core Theme 1) Concerted Action. Energy Performance of Buildings. Bruselas.

(13) Antinucci, M., Avella, G., Sentimenti, L. (2011). Inspections (Core Theme 2)-Concerted Action. Energy Performance of Buildings. Bruselas.

(14) Steering through the maze \#4. Capturing the collective knowledge base on building retrofit. European Council for an Energy Efficient Economy, 6 de mayo, 2011

(15) Low Energy Buildings In Europe: Current State of Play, Definitions and Best Practice. Nota Informativa, Comisión de la UE, 25 de septiembre, 2009

(16) Maldonado, E., et al. (2010). Report on the interim conclusions of the concerted action supporting transposition and implementation of the directive 2002/91/EC CA - EPBD (2007 - 2010). 
Tabla 1. Transposición de la EPBD en los Estados miembros de la UE.

\begin{tabular}{|c|c|c|c|c|c|c|}
\hline \multirow{2}{*}{\multicolumn{2}{|c|}{$\begin{array}{l}\text { Estado } \\
\text { miembro }\end{array}$}} & \multirow{2}{*}{\multicolumn{2}{|c|}{\begin{tabular}{|c|}
\multicolumn{2}{|c|}{ Ámbito } \\
Nacional|Regional \\
\end{tabular}}} & \multirow{2}{*}{ Normativa vigente } & \multirow{2}{*}{ Normativa previa } & \multirow{2}{*}{ Organismo regulador } \\
\hline & & & & & & \\
\hline \multicolumn{2}{|c|}{ Alemania } & $\mathrm{X}$ & & $\begin{array}{l}\text { Ordenanza de Ahorro Energétio (EnEV 2009), Erneuerbaren-Energien- } \\
\text { Wärmegesetz (Ley de Energías Renovables para Calefacción) }\end{array}$ & $\begin{array}{l}\text { Energieeinsparungsgesetz } \\
\text { 1976,Wärmeschutzverordnung 1977, EnEV 2002, En } \\
\text { EV 2004, EnEV } 2007\end{array}$ & \begin{tabular}{|l|} 
Ministerio Federal de \\
Transporte, Construcción \\
y Desarrollo Urbano junto \\
con el Ministerio Federal \\
de Economía y Tecnología. \\
Inspecciones: Ministerio \\
federal del Medio Ambiente, \\
Conservación y Seguridad \\
Nuclear.
\end{tabular} \\
\hline \multicolumn{2}{|c|}{ Austria } & & $x$ & $\begin{array}{l}\text { Ley de certificación energética (2006), Ley de certificados de eficiencia } \\
\text { energética BGBI. I No 137/2006 }\end{array}$ & $\begin{array}{l}\text { Códigos de construcción y reglamentos propios para } \\
\text { cada uno de los } 9 \text { Länders. Energieausweis-Vorlage- } \\
\text { Gesetz (EAV-G) }\end{array}$ & \begin{tabular}{|l|} 
Länder Federales con \\
Ministerio de Economía, \\
Familia y Juventud. El \\
proceso de armonización en \\
los Länder e implementación \\
lo dirige el OIB (Austrian \\
Institute of Construction \\
Engineering)
\end{tabular} \\
\hline \multirow{3}{*}{ Bélgica } & $\begin{array}{c}\text { Bruselas } \\
\text { Capital }\end{array}$ & & $x$ & $\begin{array}{l}\text { Ordenanza relativa a la eficiencia energética y temperatura interior } \\
\text { de edificios (07 junio 2007), complementada con Ordenanza de } \\
\text { eficiencia energética y calidad del aire interior (14 mayo 2009). Orden } \\
\text { de gobierno de la Región de Bruselas Capital (5 mayo 2011). Orden de } \\
\text { ejecución } 2 \text { diciembre } 2007 \text {. Orden de ejecución de } 27 \text { de mayo de } \\
\text { 2010. Orden de ejecución } 3 \text { de junio de } 2010 .\end{array}$ & - & \begin{tabular}{|l|} 
Ministerio de Medio \\
Ambiente, Energía, \\
Jurisdicción del agua y \\
Rejuvenecimiento de la \\
Ciudad.IBGE/BIM (Brussels \\
Environment Agency) y ABEA \\
(Brussels Energy Agency). \\
\end{tabular} \\
\hline & Flandes & & $X$ & Orden de ejecución de 11 mayo 2005 aprobada el 20 marzo 2009. & $\begin{array}{l}\text { Orden Ministerial relativa a la equivalencia de } \\
\text { sistemas y tecnología innovadoras (10 abril 2007), } \\
\text { Orden de Ejecución de } 1 \text { de Junio de } 1995\end{array}$ & $\begin{array}{l}\text { Ministerio de Medio } \\
\text { Ambiente, Naturaleza y } \\
\text { Energía y VEA }\end{array}$ \\
\hline & Valonia & & $x$ & $\begin{array}{l}\text { Decreto } 19 \text { abril } 2007 \text {, Orden de ejecución de } 26 \text { de Junio de } 2008 \\
\text { y refundición de } 18 \text { de Junio de } 2009 \text {. Orden de ejecución de } 3 \text { de } \\
\text { Diciembre de } 2009 \text {, y modificación de } 27 \text { de Mayo de } 2010 \text {. Orden } \\
\text { de ejecución } 17 \text { de Abril } 2008 \text {. Orden de ejecución de } 29 \text { de Enero de } \\
\text { 2009. Orden de ejecución de } 12 \text { de Julio de } 2007 .\end{array}$ & $\begin{array}{l}\text { CWATUP (Code Wallon d'Aménagement du Territoire, } \\
\text { de l'Urbanisme et du Patrimoine). } \\
\text { Reglamentos Térmicos }\end{array}$ & $\begin{array}{l}\text { Ministerio Regional de } \\
\text { Energía y Construcción } \\
\text { Sostenible y Ministerio } \\
\text { Regional de Medio Ambiente. }\end{array}$ \\
\hline \multicolumn{2}{|c|}{ Bulgaria } & $\mathrm{X}$ & & $\begin{array}{l}\text { Norma de Eficiencia Energética mediante el Decreto 54/2004, } \\
\text { enmendada en Noviembre de } 2008 \text { y Enero de 2009. Ordenanzas } \\
\text { relativas a certificación energética e inspecciones. (Varios). Reglamento } \\
\text { 16-1238. Ley de Productos Ténicos. }\end{array}$ & Requisitos Térmicos de Construcción desde 1964. & $\begin{array}{l}\text { Ministerio de Energía, } \\
\text { Economía y Turismo, Agencia } \\
\text { de Eficiencia Energética, } \\
\text { Ministerio de Desarrollo } \\
\text { Regional y Obras Públicas }\end{array}$ \\
\hline \multicolumn{2}{|c|}{ Chipre } & $\mathrm{X}$ & & $\begin{array}{l}\text { Ley Nacional para la regulación de la Eficiencia Energética de Edificios } \\
\text { N.142 (I)/2006 y enminenda N. 30(I)/2009, Reglamento 164/2009 de } \\
\text { Certificación Energética de Edificios, Reglamento } K . \Delta . \Pi \text {. 429/2006, } \\
\text { Reglamento 163/2009, Orden Ministerial 414/2009 (Metodología), } \\
\text { Orden Ministerial 446/2009 (Requisitos mínimos), Orden Ministerial } \\
\text { 260/2009 (Expertos independientes), Orden Minsiterial 413/2009 } \\
\text { (inspecciones de aire acondicionado).Orden Ministerial 412/2009 } \\
\text { (Certificados). }\end{array}$ & - & $\begin{array}{l}\text { Ministerio de Comercio, } \\
\text { Industria y Turismo } \\
\text { (Departamento de Servicios } \\
\text { Energéticos) }\end{array}$ \\
\hline \multicolumn{2}{|c|}{ Dinamarca } & $\mathrm{X}$ & & $\begin{array}{l}\text { Reglamento de Construcción Danés (BR 10), BJ431 y BJ432 la } \\
\text { Orden N. } .^{\circ} 540 \text { el cambio del etiquetado energético de los edificios } \\
\text { 05/25/2011, Orden N. }{ }^{\circ} 61 \text { de } 27 \text { Enero } 2011 \text { sobre el etiquetado } \\
\text { energético de los edificios. Consolidación de la Ley N. } 646 \text { de } \\
\text { 16/06/2011. Decreto n. }{ }^{\circ} 438 \text { de } 3 \text { de Junio } 2008 \text { (Inspecciones de } \\
\text { calderas y aires acondicionados). Decreto } 1104 \text { del 20-07-2008 }\end{array}$ & $\begin{array}{l}\text { Nuevo reglamento de energía en la construcción de } \\
1995 \text { (BR95) y construcción de viviendas de } 1998 \\
\text { (BR98). Ley del Parlamento Danés No. } 585 \text { de } 24 \\
\text { junio } 2005 \text { de ahorro energético en edificios. Decreto } \\
\text { no. } 1294 \text { de } 13 \text { diciembre } 2005 \text { de etiquetado } \\
\text { energético en edificios. Ajustes en el Decreto no. } \\
218 \text { de } 20 \text { de marzo } 2006 \text { y Decreto no. } 339 \text { de } 19 \\
\text { abril } 2006 . \text { Ley no. } 308 \text { de } 30 \text { abril de } 2008 \text {, Ley } \\
\text { N. }{ }^{\circ} 1400 \text { de } 27 \text { de diciembre de } 2008 \text {. Ley N. } 541 \\
\text { de } 26 \text { de Mayo de } 2010 \text {. Orden N. }{ }^{\circ} 1018 \text { de } 24 \text { de } \\
\text { Octubre } 2008\end{array}$ & $\begin{array}{l}\text { Agencia Danesa de Energía } \\
\text { (DEA), Danish Enterprise } \\
\text { and Construction Authoriy } \\
\text { (DEACA), Secretaría de } \\
\text { eficiencia energética en la } \\
\text { edificación (Energieffektive } \\
\text { Bygninger, SEEB) }\end{array}$ \\
\hline \multicolumn{2}{|c|}{ Eslovaquia } & $\mathrm{X}$ & & $\begin{array}{l}\text { Ley No. 555/2005 Coll, con orden de ejecución N.. }{ }^{\circ} .625 / 2006 \text { Coll. } \\
\text { Decreto N.‥311/2009 (método de cálculo). Ley n. } 17 / 2007 \text {. Decreto } \\
\text { N. }{ }^{\circ} \text { 548/2008. Decreto No 195/2008. }\end{array}$ & STN 73 0540. Ley n. ${ }^{\circ}$ 455/1991 & $\begin{array}{l}\text { Ministerio de Transporte, } \\
\text { Construcción y Desarrollo } \\
\text { Regional y el Ministerio de } \\
\text { Economía. SIEA }\end{array}$ \\
\hline \multicolumn{2}{|c|}{ Eslovenia } & $\mathrm{X}$ & & $\begin{array}{l}\text { Ley de Construcción (Nr. 102/04, } 21 \text { septiembre 2004), Ley de Energía } \\
\text { (17 noviembre 2006) y su enmienda (Nr. 27/07, } 26 \text { marzo 2007). } \\
\text { Reglamento de uso eficiente de la energía en los edificios (julio } \\
\text { 2010), Reglamento de metodología de estudios de viabilidad de sistemas } \\
\text { de energía alternativos (AES) para edificios (Abril 2009). Reglamento de } \\
\text { certificación energética de edificios, Requisitos mínimos de los sistemas } \\
\text { en PURES-2 (Julio 2010) y TSG-1-004:2010. Decreto n. }{ }^{\circ} 105-5223 / 2007 \\
\text { de } 19 \text { de Noviembre 2007 y Decreto n. }{ }^{\circ} 26 / 2008 \text { de } 17 \text { de marzo } 2008 \text {. }\end{array}$ & $\begin{array}{l}\text { Reglamento de Uso Eficiente de la Energía en los } \\
\text { Edificios (septiembre 2008), Ley de Protección del } \\
\text { Medio Ambiente (Nr. 41/04, } 22 \text { abril 2004). }\end{array}$ & $\begin{array}{l}\text { Ministerio de Energía } \\
\text { y Ministerio de Medio } \\
\text { Ambiente y Planeamiento } \\
\text { Espacial }\end{array}$ \\
\hline \multicolumn{2}{|c|}{ España } & & $x$ & \begin{tabular}{|l|} 
Real Decreto $47 / 2007$, de 19 de enero, por el que se aprueba el \\
Procedimiento básico para la certificación de eficiencia energética \\
de edificios de nueva construcción, Real Decreto $314 / 2006$, de 17 de \\
marzo, por el que se aprueba el Código Técnico de la Edificación. Real \\
Decreto $1826 / 2009$, de 27 de noviembre, por el que se modifica el \\
Reglamento de instalaciones térmicas en los edificios, aprobado por \\
Real Decreto $1027 / 2007$, de 20 de julio. Ley General para la Defensa \\
de los Consumidores y Usuarios $26 / 1984$ de 19 de julio refundida en el \\
Real Decreto Legislativo $1 / 2007$, de 16 de noviembre. Ley 38/1999, de \\
5 de noviembre, de Ordenación de la Edificación.
\end{tabular} & $\begin{array}{l}\text { NBE-CT-79: Condiciones Térmicas en los Edificio, } \\
\text { Reglamento de Instalaciones Térmicas en los Edificios. } \\
\text { RITE, aprobado por Real Decreto } 1751 / 1998, \text { de } 31 \\
\text { de julio. Real Decreto } 1027 / 2007 \text {, de } 20 \text { de julio, } \\
\text { por el que se aprueba el Reglamento de Instalaciones } \\
\text { Térmicas en los Edificios }\end{array}$ & $\begin{array}{l}\text { Ministerio de Industria, } \\
\text { Turismo y Comercio y } \\
\text { Ministerio de Fomento } \\
\text { (Secretaría de Estado de } \\
\text { Vivienda y Actuaciones } \\
\text { Urbanas) }\end{array}$ \\
\hline \multicolumn{2}{|c|}{ Estonia } & $\mathrm{X}$ & & $\begin{array}{l}\text { Reglamento N. } .^{\circ} 258 \text {, aprobado el } 20 \text { diciembre } 2007 \text {, Ley de la } \\
\text { construcción «Ehitusseaduse» N. } 107 \text { aprobada } 17 \text { diciembre } 2008 . \\
\text { Ley N. }{ }^{\circ} 146 \text { aprobada } 27 \text { agosto } 2009 .\end{array}$ & - & $\begin{array}{l}\text { Ministerio de Asuntos } \\
\text { Económicos y Comunicación. }\end{array}$ \\
\hline \multicolumn{2}{|c|}{ Finlandia } & $\mathrm{X}$ & & $\begin{array}{l}\text { Ley de Certificación Energética de Edificios (478/2007) actualizada en } \\
\text { (119/2011), Ley de Inspección de Instalaciones de Aire Acondicionado } \\
\text { (488/2007) enmendada en 2010, revisión de la Ley de Construcción y } \\
\text { Uso del Suelo. }\end{array}$ & Finlands National Building Code (D5) & $\begin{array}{l}\text { Ministerio de Medio } \\
\text { Ambiente en cooperación } \\
\text { con el Ministerio de Trabajo y } \\
\text { Economía }\end{array}$ \\
\hline \multicolumn{2}{|c|}{ Francia } & $\mathrm{x}$ & &  & $\begin{array}{l}\text { Reglamento de Construcción (enmendado en } 2005 \\
\text { y 2007), Reglamento térmico RT } 2000 \text { y RT2005, } \\
\text { Grenelle I. Inspecciones: Ley de } 5 \text { de julio 1996 }\end{array}$ & $\begin{array}{l}\text { Ministerio de Ecología, } \\
\text { de Energía y Desarrollo } \\
\text { Sostenible y Marino, y } \\
\text { Ministerio de Vivienda y } \\
\text { Medio Rural. }\end{array}$ \\
\hline
\end{tabular}




\begin{tabular}{|c|c|c|c|c|c|c|}
\hline \multirow{2}{*}{\multicolumn{2}{|c|}{ Estado miembro }} & \multicolumn{2}{|c|}{ Ámbito } & \multirow[b]{2}{*}{ Normativa vigente } & \multirow[b]{2}{*}{ Normativa previa } & \multirow[b]{2}{*}{ Organismo regulador } \\
\hline & & Nacion. & Region. & & & \\
\hline \multicolumn{2}{|c|}{ Grecia } & $x$ & & $\begin{array}{l}\text { Ley } 3361 \text { (19 mayo 2008). Orden de ejecución Ministerial K.En.A.K.- } \\
\text { D6/B/5825, Decisión Ministerial 407/09 Abril } 2010 \text { y Decreto presidencial } \\
\text { 100/NG177 (06 octubre 2010). Circular 2279/22 diciembre } 2010 .\end{array}$ & - & $\begin{array}{l}\text { Ministerio Medio Ambiente, } \\
\text { Energía y Cambio Climático } \\
\text { (MEECC) }\end{array}$ \\
\hline \multicolumn{2}{|c|}{ Hungría } & $x$ & & $\begin{array}{l}\text { Decreto Ministerial TNM 7/2006 (mayo 2006), Decreto Ministerial } \\
\text { n. }{ }^{0} 176 / 2008 \text { (junio 2008), Decreto Ministerial n. } 264 / 2008 \text { de } 6 \text { de } \\
\text { noviembre } 2008\end{array}$ & Reglamento de Construcción (desde 1991) & $\begin{array}{l}\text { Ministerio del Interior a } \\
\text { través de la Oficina Nacional } \\
\text { de Vivienda y Edificación. }\end{array}$ \\
\hline \multicolumn{2}{|c|}{ Irlanda } & $x$ & & $\begin{array}{l}\text { Reglamento de Eficiencia Energética en la Edificación } 2006 \text { (S.I. No. } \\
666 \text { de 2006), Enmienda al reglamento } 2008 \text { (SI No } 229 \text { de 2008), En- } \\
\text { mienda No. } 2 \text { al reglamento } 2008 \text { (SI No } 591 \text { de 2008) y Reglamento de } \\
\text { Inspección y Evaluación de Ciertas Instalaciones de Aire Acondicionado } \\
2006 \text { (S.I. No. } 346 \text { de 2006). Enmienda SI } 2592008 \text { (L) y enmienda } 556 \\
2009 \text { (F) }\end{array}$ & Irish Building Regulations & $\begin{array}{l}\text { Departamento de Medio } \\
\text { Ambiente, Patrimonio y } \\
\text { Gobierno Local y Departa- } \\
\text { mento de Comunicaciones, } \\
\text { Energía y Recursos Naturales. } \\
\text { Sustainable Energy Authority } \\
\text { of Ireland (SEAI) }\end{array}$ \\
\hline \multicolumn{2}{|c|}{ Italia } & & $x$ & $\begin{array}{l}\text { A nivel nacional: Decreto de } 19 \text { agosto de 2005. Decreto Presidencial } \\
2 \text { abril } 2009 \text { (metodología de cálculo, requisitos mínimos de eficiencia } \\
\text { energética e inspección de instalaciones de calefacción y aire acon- } \\
\text { dicionado). Decreto del Ministerio de Desarrollo Económico de } 25 \\
\text { de junio de } 2009 \text { (certificación energética de edificios). Inspecciones: } \\
\text { Decreto del Presidente de la República 59/2009, Ley Financiera 2008. A } \\
\text { nivel Regional: propias para cada Región. }\end{array}$ & Ley n. 10/1991. Normas propias de las 21 Regiones & $\begin{array}{l}\text { Ministerio de Desarrollo } \\
\text { Económico Ministerio de } \\
\text { Medio Ambiente } \\
\text { y el Ministerio de Infraestruc- } \\
\text { turas. Comité de Regiones }\end{array}$ \\
\hline \multicolumn{2}{|c|}{ Letonia } & $x$ & & 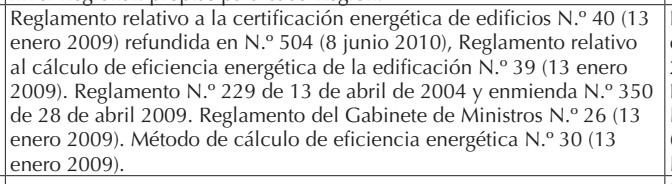 & 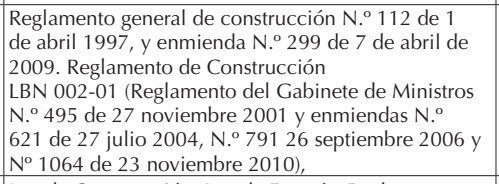 & Ministerio de Economía \\
\hline \multicolumn{2}{|c|}{ Lituania } & $\mathrm{x}$ & & $\begin{array}{l}\text { Reglamento Técnico de Construcción STR 2.01.09:2005, Orden no. } \\
\text { D-1-624. Orden No. 4-73 de } 28 \text { febrero } 2006\end{array}$ & $\begin{array}{l}\text { Ley de Construcción, Ley de Energía. Reglamento } \\
\text { Técnico de Construcción STR 1.01.09:2003 y STR } \\
\text { 2.05.01:2005 }\end{array}$ & $\begin{array}{l}\text { Ministerio de Medio Ambien- } \\
\text { te y Ministerio de Energía }\end{array}$ \\
\hline \multicolumn{2}{|c|}{ Luxemburgo } & $x$ & & $\begin{array}{l}\text { Reglamento Gran-Ducal modificado el } 30 \text { de noviembre de } 2007 \text { de } \\
\text { eficiencia energética de edificios residenciales (Memorial A n. }{ }^{\circ} 221 \\
\text { (14 diciembre 2007)), enmendada en el Memorial A n.. } 124 \text { (19 agosto } \\
2008 \text { y Memorial A n }{ }^{\circ} 9 \text { de } 21 \text { enero } 2010 \text {. Reglamento Grand-Ducal } \\
\text { de } 31 \text { de agosto de } 2010 \text { de eficiencia energética de edificios no } \\
\text { residenciales (modificado en Memorial A n }{ }^{\circ} 173 \text { de } 01 \text { octubre 2010). } \\
\text { Instalaciones: Reglamento Gran -Ducal de } 27 \text { febrero 2010, Reglamento } \\
\text { Gran -Ducal modificado el } 18 \text { abril } 2004 \text {. }\end{array}$ & \begin{tabular}{|l|} 
Ley de 5 agosto 1993 de uso racional de la energía, \\
Reglamento Gran-Ducal modificado el 23 de diciem- \\
bre 1987 relativo a las instalaciones de combustión \\
alimentadas con combustible líquido, Ley de 13 de \\
diciembre 1989 relativa a la organización de las \\
profesiones de arquitecto e ingeniero. Reglamento \\
Gran Ducal de 22 de noviembre de 1995 relativo al \\
aislamiento térmico de inmuebles \\
\end{tabular} & $\begin{array}{l}\text { Ministerio de Economía y } \\
\text { Comercio Exterior. Ministerio } \\
\text { de Desarrollo Sostenible e } \\
\text { Infraestructuras. }\end{array}$ \\
\hline \multicolumn{2}{|c|}{ Malta } & $\mathrm{x}$ & & $\begin{array}{l}\text { Reglamento de Eficiencia Energética en la Edificación LN 261/2008 } \\
\text { (Malta Resources Authority Act capitulo 423) de } 21 \text { de octubre de 2008, } \\
\text { Aviso Legal } 238 \text { (6 octubre 2006) }\end{array}$ & $\begin{array}{l}\text { Ley de eficiencia energética en edificios LN } \\
\text { 238/2006, Requisitos mínimos de eficiencia energé- } \\
\text { tica de edificios, Guía técnica Documento F (Aviso } \\
\text { N. } 1002 \text { de 2006) }\end{array}$ & $\begin{array}{l}\text { Malta Resources Authority } \\
\text { (MRA), que depende del } \\
\text { Ministerio de Recursos y } \\
\text { Asuntos Rurales. Building } \\
\text { Regulation Office (BRO) } \\
\end{array}$ \\
\hline \multicolumn{2}{|c|}{$\begin{array}{l}\text { Paises } \\
\text { Bajos }\end{array}$} & $x$ & & $\begin{array}{l}\text { Decreto de Eficiencia Energética de Edificios (BEG) de } 24 \text { noviembre de } \\
\text { 2006, Reglamento de eficiencia energética de edificios (REG) de } 29 \text { de } \\
\text { diciembre de 2006, Eficiencia energética de los edificios (BS 7120) (15 } \\
\text { abril 2011) }\end{array}$ & $\begin{array}{l}\text { REG basada en BRL9600 (ISSO } 75 \text { no residenciales y } \\
\text { ISSO } 82 \text { residenciales) y BRL9501 (ISSO 54) }\end{array}$ & $\begin{array}{l}\text { Ministerio del Interior y } \\
\text { Relaciones del Reino }\end{array}$ \\
\hline \multicolumn{2}{|c|}{ Polonia } & $x$ & & $\begin{array}{l}\text { Modificaciones a la Ley de Construcción aprobadas el } 27 \text { agosto } 2009 . \\
\text { Ordenanza de } 21 \text { de enero de } 2008 \text { sobre la formación y examen de } \\
\text { expertos, Ordenanza de } 13 \text { de noviembre de } 2008 \text { sobre la metodología } \\
\text { de cálculo de eficiencia energética y Ordenanza de } 13 \text { de noviembre } \\
\text { sobre requisitos energéticos mínimos. Enmiendas a las Ordenanzas } \\
\text { del Ministerio de Infraestructura de } 12 \text { de abril } 2002 \text { y } 3 \text { de julio } 2003 \text {. } \\
\text { Ordenanza } 21 \text { de enero de } 2008 .\end{array}$ & $\begin{array}{l}\text { Ley de Construcción, aprobada el } 19 \text { septiembre } \\
\text { 2007, Ordenanzas del Ministerio de Infraestructura } \\
\text { de } 12 \text { de abril } 2002 \text { y } 3 \text { de julio } 2003 \text {. Ley de Termo- } \\
\text { Modernización } 1978\end{array}$ & $\begin{array}{l}\text { Ministerio de Infraestructuras } \\
\text { y Ministerio de Economía }\end{array}$ \\
\hline \multicolumn{2}{|c|}{ Portugal } & $x$ & & $\begin{array}{l}\text { Decretos 78/2006, 79/2006, y 80/2006. "Portaria N.461/2007" “Portaria } \\
\text { N.835/2007", Despacho n. }{ }^{\circ} 11020 / 2009 \text {, Despacho n. }{ }^{\circ} \text { 10250/2008 }\end{array}$ & $\begin{array}{l}\text { Reglamento de la envolvente de edificios (desde } \\
\text { 1990). Reglamento de instalaciones HVAC (desde } \\
\text { 1998) }\end{array}$ & $\begin{array}{l}\text { Ministerio de Economía en } \\
\text { colaboración con el Ministe- } \\
\text { rio de Medio Ambiente }\end{array}$ \\
\hline \multirow{3}{*}{$\begin{array}{l}\text { Reino } \\
\text { Unido }\end{array}$} & Escocia & & $x$ & $\begin{array}{l}\text { The Building (Scotland) Act 2003, enmienda al Building (Scotland) } \\
\text { Regulations 2010, Building (Procedure) (Scotland) Regulations 2007, } \\
\text { the Building (Forms) (Scotland) Regulations } 2007 \text { y Energy Performance } \\
\text { of Buildings Regulations 2008. }\end{array}$ & $\begin{array}{l}\text { Building (Scotland) Regulations } 2004 \text { (enmiendas en } \\
\text { 2006, 2007, 2008) }\end{array}$ & $\begin{array}{l}\text { Building Standards Division, } \\
\text { que forma parte del Direc- } \\
\text { torate for the Built Environ- } \\
\text { ment. }\end{array}$ \\
\hline & $\begin{array}{l}\text { Inglaterra } \\
\text { y Gales }\end{array}$ & & $x$ & $\begin{array}{l}\text { Reglamento de Construcción (Statutory Instrument 2010/2214), y } \\
\text { S.I.2010/2215. }\end{array}$ & $\begin{array}{l}\text { Reglamento de Construcción S.I.2000/2531, S.I. } \\
\text { 2000/2532, S. I. 2001/3336, S. I. 2002/2872, S.I. } \\
\text { 2004/1466, S I 2008/2363. }\end{array}$ & $\begin{array}{l}\text { Departamento de Comunida- } \\
\text { des y Gobierno Local (CLG), } \\
\text { Departamento de Medio } \\
\text { Ambiente, Alimentación y } \\
\text { Asuntos Rurales (Defra) y el } \\
\text { Departamento de Energía y } \\
\text { Cambio Climático (DECC) }\end{array}$ \\
\hline & $\begin{array}{c}\text { Irlanda } \\
\text { del Norte }\end{array}$ & & $x$ & $\begin{array}{l}\text { The Building (Amendment N. }{ }^{\circ} \text { 2) Regulations (Northern Ireland) } \\
\text { 2010. SR } 2010 \mathrm{~N} .{ }^{\circ} 382 \text { (Technical Booklets: F1 dy F2), The Energy } \\
\text { Performance of Buildings (Certificates and Inspections) (Amendments) } \\
\text { Regulations (Northern Ireland) } 2009 \text { SR } 2009 \text { N. }{ }^{\circ} 369\end{array}$ & $\begin{array}{l}\text { Statutory Rule-2006 N. } 355 \text { - The Building (Amend- } \\
\text { ment) Regulations (Northern Ireland), The Building } \\
\text { (Amendment N. } .^{\circ} \text { 2 Regulations (Northern Ireland) } \\
2006^{\prime \prime} \text { SR 2006 N. }{ }^{\circ} 440 . \text { The Energy Performance of } \\
\text { Buildings (Certificates and Inspections) Regulations } \\
\text { (Northern Ireland) } 2008 \text { Statutory Rule } 2008 \text { N. } .^{\circ} 170 .\end{array}$ & $\begin{array}{l}\text { Departamento de Finanzas y } \\
\text { Personal (DFPNI) y Departa- } \\
\text { mento de Desarrollo Social } \\
\text { (DSDNI) }\end{array}$ \\
\hline \multicolumn{2}{|c|}{$\begin{array}{l}\text { República } \\
\text { Checa }\end{array}$} & $x$ & & $\begin{array}{l}\text { Enmienda a la Ley de Gestión de la Energía de } 26 \text { de marzo de } 2006 . \\
\text { Decreto N. } \text {. }^{148 / 2007} \text { acerca de la eficiencia energética de edificios. } \\
\text { Reglamento N. }{ }^{\circ} 276 / 2007 \text {. Ley N. }{ }^{\circ} 150 / 2001 \text {. CSN } 7305 \text { 040-2/Z1: } \\
2005\end{array}$ & $\begin{array}{l}\text { Ley de Gestión de la Energía. Ley 458/2000 (Ley de } \\
\text { la Energía). Ley N. } \text {. }^{\circ} 406 / 2006 \text { Coll., que contiene } \\
\text { el texto completo de la Ley N. }{ }^{\circ} \text { 406/2000 Coll. , en } \\
\text { materia de energía, en su versión modificada por la } \\
\text { Ley N. }^{\circ} \text { 359/2003 Coll., la Ley N. }{ }^{\circ} 694 / 2004 \text { Coll. } \\
\text { Ley N. }{ }^{\circ} 180 / 2005 \text { Coll. y la Ley N. }{ }^{\circ} 177 / 2006 \text { Coll. } \\
\text { CSN } 7305040 \text { (1964, 1979, 1994, 2002) } \\
\end{array}$ & $\begin{array}{l}\text { Ministerio de Industria y } \\
\text { Comercio }\end{array}$ \\
\hline \multicolumn{2}{|c|}{ Rumanía } & $x$ & & $\begin{array}{l}\text { Ley } 372 / 2005 \text { de } 19 \text { de diciembre de } 2005 \text {, Orden } 691 / 1459 / 288 / 2007 \\
\text { de } 12 \text { de octubre de } 2007 \text {, Orden } 157 / 2007 \text { de } 1 \text { de febrero de } 2007 \text {, ac- } \\
\text { tualizada en la Orden } 1071 / 2009 \text {, Orden } 2055 / 2005 \text { de } 13 \text { de diciembre } \\
\text { de } 2005 \text {, y enmienda de octubre de } 2010 \text { (C107/2010). Orden } 174 / 2008, \\
\text { Orden } 18 / 2009 \text { de } 12 \text { de marzo de } 2009 \text {. Orden } 1071 / 2009 \text { (como com- } \\
\text { plemento a la } 1057 / 2007 \text {. Orden } 69 / 2010 \text { de } 1 \text { de julio de } 2010\end{array}$ & Orden C107/1997, Orden C107/2007 & $\begin{array}{l}\text { Ministerio de Desarrollo } \\
\text { Regional y Turismo }\end{array}$ \\
\hline \multicolumn{2}{|c|}{ Suecia } & $x$ & & $\begin{array}{l}\text { Código de Construcción BBR 12,BED (BFS 2007:4), revisada en el } \\
\text { BED } 2 \text { (BFS 2007:5) y BED } 3 \text { (2010), Ley 2006:985 de Junio de } 2006 \text { y } \\
\text { Ordenanza 2006:1592 de Diciembre de 2006, Revisión de la Ordenan- } \\
\text { za (1994:1215) en } 2008 \text { y Normas de Construcción BFS 2008:20. CEX } 2 \\
\text { (2010), Ley 2006:985. }\end{array}$ & \begin{tabular}{|l|} 
BSF 2006;12, CEX, BFS 2007:5, BBR (BFS 1993:57) \\
enmienda en 2008, Ley de construcción y planea- \\
miento 1987:10 (PBL), decreto de planeamiento y \\
construcción (1987:383), Ley de requisitos técnicos \\
para construcción (1994:847) y Decreto en requisitos \\
técnicos de construcción (1994:1215), hasta BBR \\
2006:22.
\end{tabular} & $\begin{array}{l}\text { Ministerio de Empresa, Mi- } \\
\text { nisterio de Medio Ambiente. } \\
\text { Boverket }\end{array}$ \\
\hline
\end{tabular}




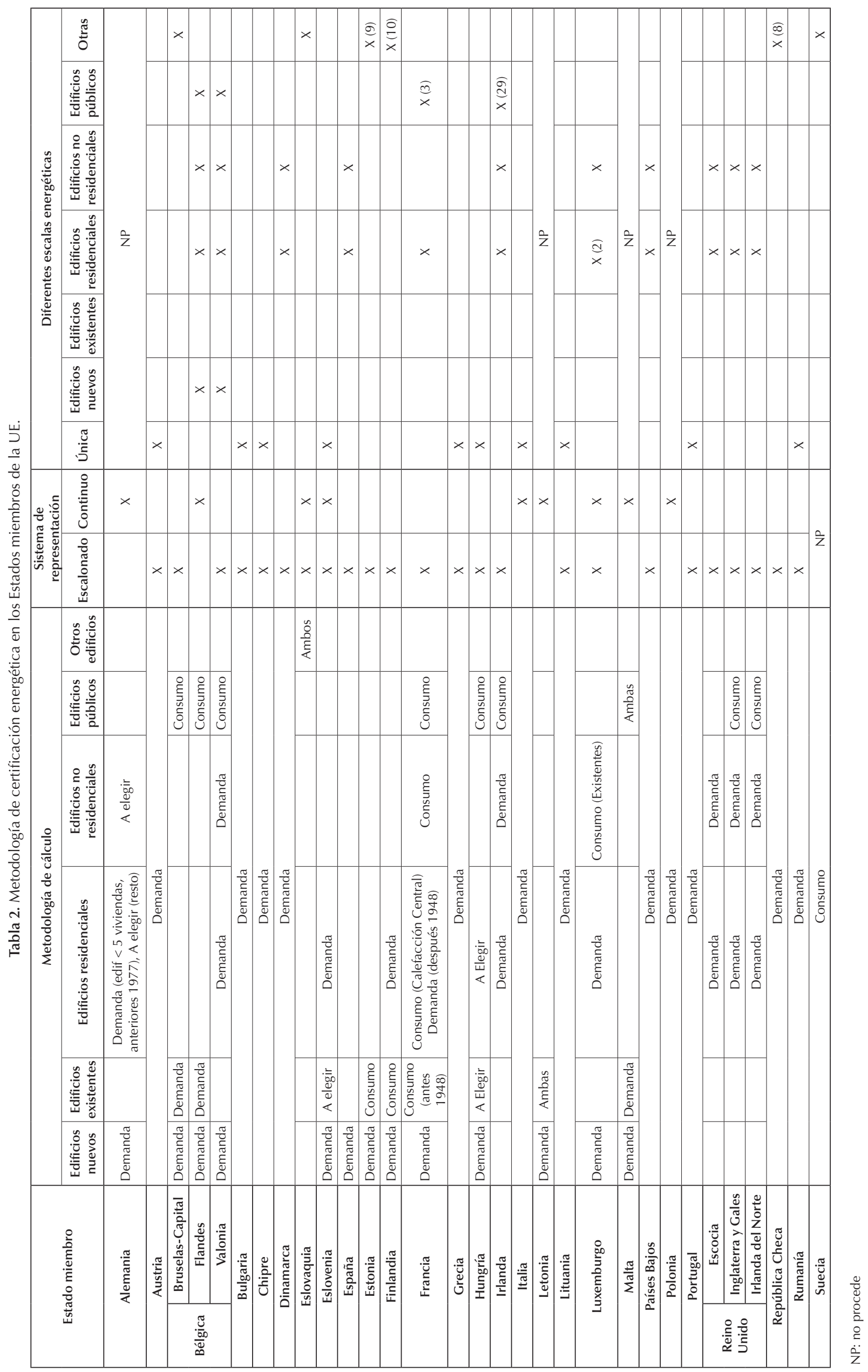

